\title{
Local Chemical and Deformation Profiles in InAs/AISb Multilayer Structures for Quantum Cascade Lasers
}

B. Warot-Fonrose ${ }^{1,4}$, J. Nicolaï ${ }^{1,4}$, M. Vallet $^{1,4}$, C. Gatel ${ }^{1,4}$, R.Teissier ${ }^{2}$, A. N. Baranov ${ }^{2}$, C. Magen $^{3,4}$ and A. Ponchet ${ }^{1,4}$

1 CEMES CNRS-UPR 8011, Université de Toulouse, 31055 Toulouse, France

2 IES CNRS-UMR 5214, 34095 Montpellier, France

3 Laboratorio de Microscopías Avanzadas (LMA), Instituto de Nanociencia de Aragón (INA) ARAID and Departamento de Física de la Materia Condensada, Universidad de Zaragoza, 50018 Zaragoza, Spain

4 Transpyrenean Associated Laboratory for Electron Microscopy (TALEM), CEMES-INA, CNRS-Universidad de Zaragoza

Optoelectronic devices are widely studied to master the growth process of the semiconductors and achieve the best performances. Some of these systems have very large conduction band offsets (OBC), which make them attractive for use in devices using the in-band electronic transitions . For example, the multilayer InAs / AlSb deposited on InAs has been used with success to achieve quantum cascade lasers (QCL) for emitting short wavelength below $3 \mu \mathrm{m}$ [1].

These Quantum Cascade Lasers are the result of a complex architecture with many semiconducting layers deposited as very thin films. Any defect or strain accumulation could lead to the loss of optical properties. Among various structural parameters, the determination of the deformations at the local level is crucial. Interfaces are especially of major interest as they concentrate the chemical / structural transitions. In InAs/AlSb samples, the absence of any common atom between the barrier and the well induces that new bonds need to be formed. Depending on the AlAs or InSb type, compression or tension will be experienced by the interface. Local chemistry and strain state are therefore intimately linked and this work proposes to combine these two measurements on this technologically interesting system.

The deformations are determined on a $\mathrm{C}_{\mathrm{s}}$ corrected Tecnai microscope in high resolution mode. GPA analysis allows for the determination of deformation profiles across the layers and the interfaces. Special care has been taken to select proper images and imaging conditions to apply the treatment and an extensive study of the influence of the mask size used has been made.

Combined with the deformation measurements, STEM-HAADF images have been acquired on a probe $\mathrm{C}_{\mathrm{s}}$ corrected Titan (INA Zaragoza) to quantify the intensity variation the vicinity of the interfaces. The EELS signal has also been recorded and correlated with HAADF images intensity.

Several samples have been studied with specific interface preparation. The sequence of the opening of the different cells in the MBE chamber has been tuned to favor either compressive or tensile deformation at the interfaces. Considering local strain and chemistry, we estimated the interface composition and discussed the mechanisms of interface formation for the different growth sequences. One example is shown on figure 1c, where the deformation profile extracted from the deformation map is plotted. The tensile stress at both interfaces is clearly visible. Combined with STEM-HAADF image intensity analysis (figure 2), we found therefore that formation of the AlAs-type interface is spontaneously favored, due to the very high thermal 
stability compared to the InSb-type interface. We also showed that the interface composition could be tuned using an appropriate growth sequence [2,3].

\section{References}

[1] J. Devenson et al, Applied Physics Letters, 91, (2007), 141106

[2] J. Nicolai et al, Applied Physics Letters, 104, (2014), 031907

[3] Acknowledgements: The authors are grateful to C. Crestou for TEM samples thinning. This work is supported by the French national project NAIADE (ANR-11-BS10-017) and the European Union Seventh Framework Programme under Grant Agreement 312483 - ESTEEM2 (Integrated Infrastructure Initiative-I3).
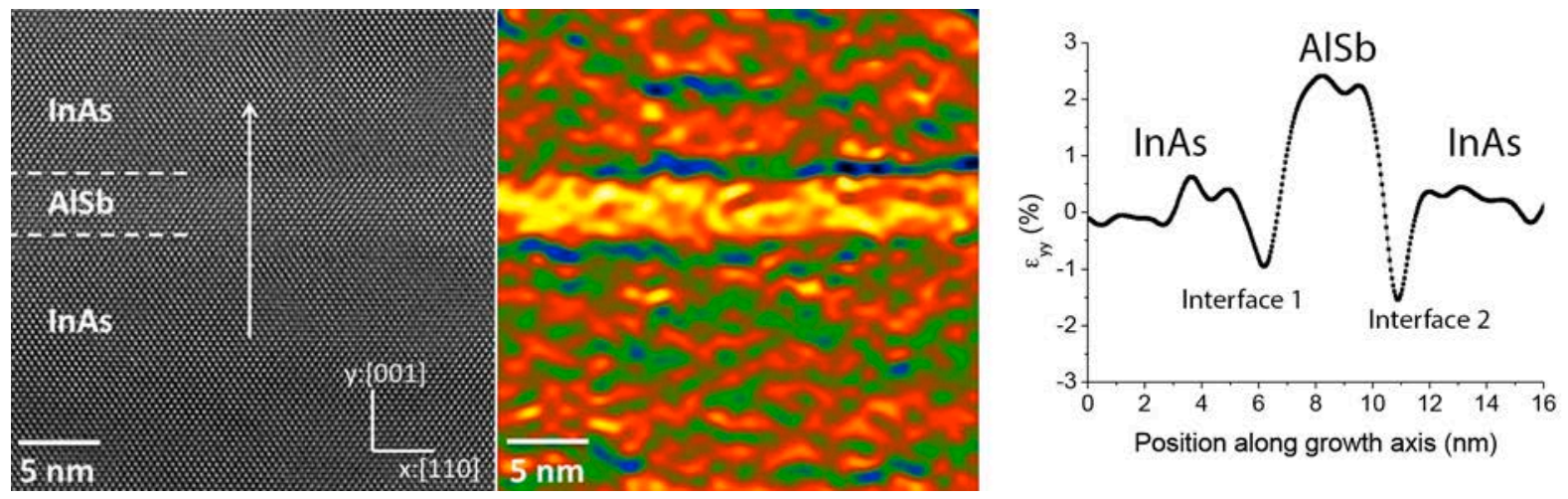

Figure 1. a) HREM image of a InAs/AlSb/InAs sample, the white arrow indicates the growth direction, b) mapping of the deformation of the planes parallel to the interface, c) deformation profile obtain by the GPA method average on the whole width of the image (30 nm)
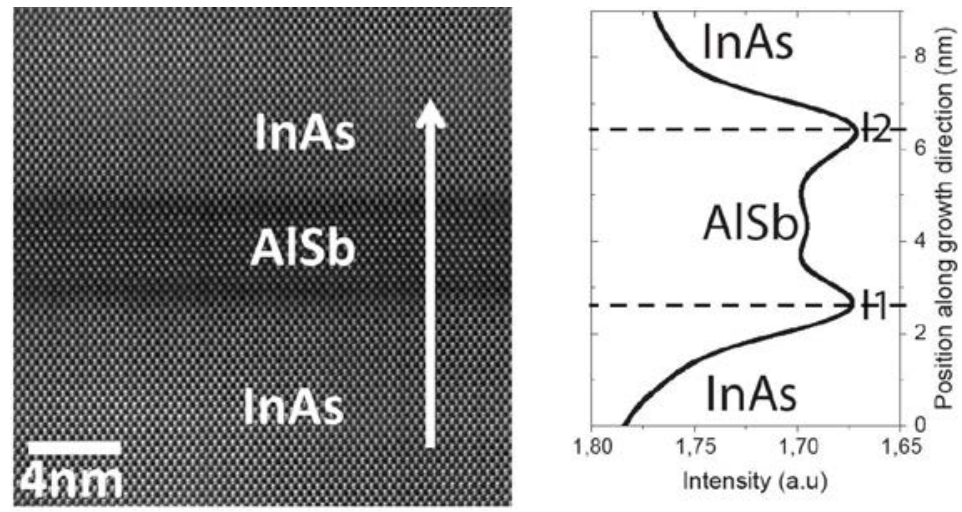

Figure 2. STEM-HAADF image of the same sample as the one presented on figure 1 (left), intensity profile along the growth direction 